\title{
Phase portrait of a matter bounce in Hořava-Lifshitz cosmology
}

\author{
E. Czuchry \\ ${ }^{1}$ Instytut Problemów Jạdrowych, ul. Hoża 69, 00-681 Warszawa, Poland \\ E-mail: eczuchry@fuw.edu.pl
}

\begin{abstract}
.
The occurrence of a bounce in FRW cosmology requires modifications of general relativity. An example of such a modification is the recently proposed Hořava-Lifshitz theory of gravity, which includes a "dark radiation" term with a negative coefficient in the analog of the Friedmann equation. This letter describes a phase space analysis of models of this sort with the aim of determining to what extent bouncing solutions can occur. It is found that they are is possible, but not generic in models under consideration. Apart from previously known bouncing solutions some new ones are also described. Other interesting solutions found include ones which describe a novel sort of quasi stationary, oscillating universes.
\end{abstract}


Phase portrait of a matter bounce in Horava-Lifshitz cosmology

\section{Introduction}

The standard $\Lambda$ CDM model has solved many issues in cosmology. However, in spite of all this success, it also leaves a number of issues unaddressed. Perhaps the most significant is the problem of initial singularity, where general relativity breaks down. There have been many attempts to modify Einstein's theory to avoid this singularity. Some are made at classical level, some involve quantum effects. Examples include the ekpyrotic/cyclic model ([1, 2, 3]) and loop quantum cosmology $(4,5,6])$, which replace the Big Bang with a Big Bounce. Attempts to address these issues at the classical level include braneworld scenarios ( 7 , 8]), where the universe goes from an era of accelerated collapse to an expanding era without any divergences or singular behavior. There are also higher order gravitational theories and theories with scalar fields (see 9] for a review of bouncing cosmologies). It is however fair to say that the issue of the initial singularity remains one of the key questions of early Universe cosmology, and the idea that it is avoided due to a bounce is remains an elusive (and promising) notion. As discussed below, it is clear that close to the singularity the Friedmann equation has to be modified for a bounce to be possible.

In recent months much effort has been devoted to studies of a proposal for a UV complete theory of gravity due to Hořava [10, 11, 12, 13]. Because in the UV the theory possesses a fixed point with an anisotropic, Lifshitz scaling between time and space, this theory is referred to as Hořava-Lifshitz gravity. Soon after this theory was proposed many specific solutions of this theory have been found, including cosmological ones ([14, 15, 16, 17, 18, 19, 20, 21]). It was also realized that the analog of the Friedmann equation in HL gravity contains a term which scales in the same way as dark radiation in braneworld scenarios [14, 15, 16] and gives a negative contribution to the energy density. Thus, at least in principle it is possible to obtain non-singular cosmological evolution within Hořava theory, as it was pointed out in 14, 16, 22.

Although there is presently much discussion of possible problems and instabilities of HoravaLifshitz gravity [23, 24, 25, 26], it is still very interesting to perform a detailed investigation of the influence of the additional terms in the Friedmann equation in HL gravity on the existence and stability of a cosmological bounce. In this paper we are going to analyze how these terms affect the dynamics of the system using phase portrait techniques described in [32, 33, and then compare the results with those valid in standard cosmology. For purpose of illustration we will assume that matter in the pre-bounce epoch is described by a scalar field $\varphi$ with a quadratic potential. In order to concentrate on modifications created by the "dark radiation" terms, we will set cosmological constant $\Lambda=0$. Such scenario may be also considered as an approximation to a general case with non-vanishing $\Lambda$, valid in the regime of small scale factor $a$, when standard curvature and cosmological constant terms (vanishing for $\Lambda=0$ ) become negligible. Thus, the present analysis can be regarded as an exploration of the cosmologies with modified equations of motion, where the particular modifications considered are inspired by Hořava cosmology. In particular, the main question addressed is the impact of these modifications on the existence of a bounce, which is otherwise not possible.

Related analysis of Hořava-Lifshitz cosmology have recently appeared in 27] and [28, which we become aware of while this work was being typed. Those papers address a somewhat different set of issues from what we have pursued. The analysis presented in [27] and 28] consider the full 4-dimensional phase space of HL cosmology. The results presented here focus on the region close to where the scale factor vanishes, which admits a critical simplification: the number of dynamical equations under study can be reduced from 4 to 3 (as discussed in more detail in Section 3). This makes it possible to visualize the possible phase space trajectories in a 3-dimensional space.

The structure of this note is following: in Section 2. we briefly sketch Hořava-Lifshitz gravity and cosmology. In Section 3. the possibility of bounce is discussed. In Section 4 we discuss phase portraits of the discussed system of equations and describe different families of phase trajectories. 


\section{Hořava-Lifshitz cosmology}

The metric of Hořava-Lifshitz theory, due to anisotropy in UV, is written in the (3+1)-dimensional ADM formalism:

$$
d s^{2}=-N^{2} d t^{2}+g_{i j}\left(d x^{i}-N^{i} d t\right)\left(d x^{j}-N^{j} d t\right),
$$

where $N, N_{i}$ and $g_{i j}$ are dynamical variables. The action of Hořava-Lifshitz theory is [12]

$$
\begin{aligned}
& I=\int d t d^{3} x\left(\mathcal{L}_{0}+\mathcal{L}_{1}\right), \\
& \mathcal{L}_{0}=\sqrt{g} N\left\{\frac{2}{\kappa^{2}}\left(K_{i j} K^{i j}-\lambda K^{2}\right)+\frac{\kappa^{2} \mu^{2}\left(\Lambda R-3 \Lambda^{2}\right)}{8(1-3 \lambda)}\right\}, \\
& \mathcal{L}_{1}=\sqrt{g} N\left\{\frac{\kappa^{2} \mu^{2}(1-4 \lambda)}{32(1-3 \lambda)} R^{2}-\frac{\kappa^{2}}{2 \omega^{4}} Z_{i j} Z^{i j}\right\},
\end{aligned}
$$

where $K_{i j}=\frac{1}{N}\left[\frac{1}{2} \dot{g}_{i j}-\nabla_{(i} N_{j)}\right]$ is extrinsic curvature of a spacelike hypersurface with a fixed time, a dot denotes a derivative with respect to $t$ and covariant derivatives defined with respect to the spatial metric $g_{i j}$. Moreover

$$
Z_{i j}=C_{i j}-\frac{\mu \omega^{2}}{2} R_{i j}
$$

$\kappa^{2}, \lambda, \mu, \omega$ and $\Lambda$ are constant parameters and the Cotton tensor, $C_{i j}$, is defined by

$$
C^{i j}=\epsilon^{i k l} \nabla_{k}\left(R_{l}^{j}-\frac{1}{4} R \delta_{l}^{j}\right)=\epsilon^{i k l} \nabla_{k} R_{l}^{j}-\frac{1}{4} \epsilon^{i k j} \partial_{k} R .
$$

In (2), $\mathcal{L}_{0}$ is the kinetic part of the action, while $\mathcal{L}_{1}$ gives the potential of the theory in the so-called "detailed-balance" form.

Matter may be added by introducing a scalar field $\varphi([14,15])$ with energy density $\rho$ and pressure $p$. The action for matter is

$$
I_{m}=\int d t d^{3} x \sqrt{g} N \mathcal{L}_{m}
$$

The matter Lagrangian $\mathcal{L}_{m}$ depends on the scalar matter field $\varphi$ and the 4-dimensional metric. $\mathcal{L}_{m}:$

$$
\mathcal{L}_{m}=\frac{3 \lambda-1}{2}\left(\frac{1}{2 N^{2}}\left(\dot{\varphi}^{2}-N^{i} \partial_{i} \varphi\right)-V(\varphi)\right)
$$

This allows to define the energy density and pressure of the scalar field in the following way:

$$
\begin{aligned}
& \rho=\frac{3 \lambda-1}{4} \dot{\varphi}^{2}+V(\varphi), \\
& p=\frac{3 \lambda-1}{4} \dot{\varphi}^{2}-V(\varphi)
\end{aligned}
$$

In numerical calculations presented further on a specific form of the scalar potential will be assumed (see eqn. (14)).

Comparing the action of Hořava-Lifshitz theory to the Einstein-Hilbert action of general relativity, one can see that the speed of light, Newton's constant and the cosmological constant are

$$
c=\frac{\kappa^{2} \mu}{4} \sqrt{\frac{\Lambda}{1-3 \lambda}}, \quad G=\frac{\kappa^{2} c}{32 \pi}, \quad \Lambda_{E}=-\frac{3 \kappa^{4} \mu^{2}}{3 \lambda-1} \frac{\Lambda^{2}}{32},
$$

respectively. Setting dynamical constant $\lambda=1$, reduces the first three terms in (2) to the usual ones of Einstein's relativity and matter Langrangian in (5) to the usual scalar field action in curved space-time.

The equations for Hořava-Lifshitz cosmology are obtained by imposing condition of homogeneity and isotropy of the metric. Precisely, the equations of motion are obtained by varying 
the action (2) with respect to $N, a$, and $\varphi$, and setting $N=1$ at the end of the calculation, leading to

$$
\begin{aligned}
& H^{2}=\frac{\kappa^{2} \rho}{6(3 \lambda-1)}+\frac{\kappa^{4} \mu^{2} \Lambda}{8(3 \lambda-1)^{2}} \frac{k}{a^{2}}-\frac{\kappa^{4} \mu^{2}}{16(3 \lambda-1)^{2}}\left(\Lambda^{2}+\frac{k^{2}}{a^{4}}\right), \\
& \dot{H}=-\frac{\kappa^{2}(\rho+p)}{4(3 \lambda-1)}-\frac{\kappa^{4} \mu^{2} \Lambda}{8(3 \lambda-1)^{2}} \frac{k}{a^{2}}+\frac{\kappa^{4} \mu^{2}}{32(3 \lambda-1)^{2}} \frac{k^{2}}{a^{4}},
\end{aligned}
$$

and also equation of motion for the scalar field:

$$
\ddot{\varphi}+3 H \dot{\varphi}+\frac{2}{3 \lambda-1} V^{\prime}=0,
$$

where $H=\dot{a} / a$, a prime denotes the derivative with respect to scalar field $\varphi$. The significant new terms in the above equations of motion are the $\left(1 / a^{4}\right)$-terms on the right-hand sides of 10 . and (11). They are reminiscent of the dark radiation term in braneworld cosmology [29] and are present only if the spatial curvature of the metric is non-vanishing.

Equations 10 and (11) show different behavior for different ranges of the $\lambda$-parameter: $\lambda>1 / 3, \lambda=1 / 3$ and $\lambda<1 / 3$. It was shown in 30. that $1>\lambda>1 / 3$ leads to ghost instabilities in the IR limit of the theory. Solution to this problem proposed in 31 results in instabilities re-emerging at UV region. However, this range of $\lambda$ is exactly the flow-interval between the UV and IR regimes. Thus the only physically interesting case that remains, allowing for a possible flow towards GR - at $\lambda=1-$ is the regime $\lambda \geq 1$. Region $\lambda \leq 1 / 3$ is disconnected from $\lambda=1$ and cannot be included in realistic considerations.

Thus we will remain in the phenomenologically range relevant $\infty>\lambda \geq 1$. In this case the value of $\lambda$-a dimensionless coupling of the theory - may be included in rescalling of the parameter $\kappa$. In general, $\lambda$ runs - logarithmically in the UV - and may eventually reach one the three IR fixed points ([1]): $\lambda=1 / 3, \lambda=1$ or $\lambda=\infty$, the first one excluded by the existence of instabilities.

\section{Existence of bounce}

New terms in the cosmological equations introduce the possibility of a bounce. The form of (10), with $k= \pm 1$ implies that it is possible that $H=0$ at some moment of time. This is a necessary condition for the realization of the bounce. It was pointed out in [14, that it may happen in the presence of matter, at the critical time $t_{*}, a=a_{*}$, when the critical energy density is equal to

$$
\rho=\rho_{*}=\frac{3 \kappa^{2} \mu^{2}}{3 \lambda-1}\left(-\frac{\Lambda}{4} \frac{k}{a_{*}^{2}}+\frac{\Lambda^{2}}{8}+\frac{1}{8} \frac{k^{2}}{a_{*}^{4}}\right),
$$

which is determined by the couplings of the theory.

From the continuity equation it follows that at the bounce point $\dot{H}>0$. Therefore a transition from a contracting to an expanding phase may be possible. It was shown in 22 that the necessary condition for a cosmological bounce is that the energy density of regular matter increases less fast than $a^{-4}$ as the scale factor decreases and $\left(\frac{\rho}{12}-p\right)>0$.

We begin our considerations during a contracting phase. At the beginning the scale factor is quite large and the contribution of dark radiation to the total energy density is quite small. As the universe contracts, the energy density increases and the scale factor decreases rapidly. When a critical density is achieved, a big bounce is about to take place.

One would expect that near the bounce the leading term in 10 and 11 would be the dark radiation one, with curvature and cosmological constant terms neglectable small. Actually the latter terms generally vanish when HL cosmological constant $\Lambda=0$. In case of non-vanishing $\Lambda$ these two terms may be neglected when the scale factor $a$ is sufficiently small. Specifically, assuming for a moment an equation of state of the form $p=w \rho$ with constant $w$, it is well known that $H^{2}, \dot{H}$ and $\rho$ scale as $a^{-3(1+w)}$. Therefore we may keep the density term and omit the curvature term $\sim 1 / a^{2}$ if $w>-\frac{1}{3}$. In the case of a quadratic potential considered below (for which $w \neq$ const, so the above argument does not directly apply) we have checked numerically that in all bounce scenarios discussed in this paper, this approximation is valid near bounce point (up to $10^{-7}$ ). 
We will model the matter sector in this pre-bounce epoch by assuming it is described by a scalar field $\varphi$ with a potential

$$
V(\varphi)=\frac{1}{2} m^{2} \varphi^{2}
$$

For calculational simplicity we put $m=1$.

This way and inserting $\alpha=2 / \kappa^{2}$, we have the following equations modeling bounce in the Hořava-Lifshitz cosmology.

$$
\begin{aligned}
\dot{H} & =-\frac{\kappa^{2}}{4(3 \lambda-1)} \dot{\varphi}^{2}+\frac{\kappa^{4} \mu^{2}}{8(3 \lambda-1)^{2}} \frac{k^{2}}{a^{4}}, \\
H^{2} & =\frac{\kappa^{2}}{12(3 \lambda-1)}\left(\dot{\varphi}^{2}+\varphi^{2}\right)-\frac{\kappa^{4} \mu^{2}}{16(3 \lambda-1)^{2}} \frac{k^{2}}{a^{4}} .
\end{aligned}
$$

The value of $\kappa^{2}$ may be expressed in terms of cosmological constants $(9)$, we will work in units such that $8 \pi G=1$ and $c=1$. Then

$$
\kappa^{2}=32 \pi G c
$$

and the values of $\mu$ are left arbitrary. Therefore the Friedmann equations take the following form near the bounce:

$$
\begin{aligned}
\dot{H} & =-\frac{1}{3 \lambda-1} \dot{\varphi}^{2}+\frac{2 \mu^{2} k^{2}}{(3 \lambda-1)^{2} a^{4}} \\
H^{2} & =\frac{1}{3(3 \lambda-1)}\left(\dot{\varphi}^{2}+\varphi^{2}\right)-\frac{\mu^{2} k^{2}}{(3 \lambda-1)^{2} a^{4}},
\end{aligned}
$$

Additionally, completing dynamics of the system, there is the equation of motion for the scalar field and the definition of the Hubble parameter:

$$
\begin{aligned}
& \ddot{\varphi}=-\frac{2}{3 \lambda-1} \varphi-3 \dot{\varphi} H, \\
& \dot{a}=a H .
\end{aligned}
$$

The value of the parameter $\mu$ may be kept arbitrary. This parameter does not alter solutions of the system (18,21, but it specifies values of $a$ on an obtained trajectory.

\section{Phase portrait}

\subsection{Phase space}

The local geometry of the phase portrait is characterized by the nature and position of its critical points. These points are locations where the derivatives of all the dynamic variables, i.e. the r.h.s. of (33 35), vanish. Moreover, they are the only points where phase trajectories may start, end, or intersect. They can also begin or end in infinity, and then - after a suitable coordinate transformation projecting the complete phase space onto a compact region (so called Poincaré projection) - there may be well defined infinite critical points. The set of finite and infinite critical points and their characteristic, given by the properties of the Jacobian matrix of the linearized equations at those points, provides a qualitative description of the given dynamical system.

Dynamics of our system is described by the following set of first order ODE's:

$$
\begin{aligned}
u & =\dot{\varphi} \\
\dot{u} & =-\frac{2}{3 \lambda-1} \varphi-3 u H, \\
\dot{a} & =a H \\
\dot{H} & =-\frac{1}{3 \lambda-1} u^{2}+\frac{2 \mu^{2} k^{2}}{(3 \lambda-1)^{2} a^{4}}
\end{aligned}
$$

plus the constraint equation:

$$
H^{2}=\frac{1}{3(3 \lambda-1)}\left(u^{2}+\varphi^{2}\right)-\frac{\mu^{2} k^{2}}{(3 \lambda-1)^{2} a^{4}}
$$


If spatial curvature $k=0$ one may consider a 2-dimensional subsystem:

$$
\begin{aligned}
u & =\dot{\varphi} \\
\dot{u} & =-\frac{2}{3 \lambda-1} \varphi-3 u H, \\
\dot{H} & =-\frac{1}{3 \lambda-1} u^{2}
\end{aligned}
$$

with a constraint equation

$$
H^{2}=\frac{1}{3(3 \lambda-1)}\left(u^{2}+\varphi^{2}\right)
$$

If $k \neq 0$ one may also consider a subsystem on variables $(\varphi, u, H)$, obtained via reduction of the original system with respect to constraint 26. Namely, substituting

$$
\frac{\mu^{2} k^{2}}{(3 \lambda-1)^{2} a^{4}}=\frac{1}{3(3 \lambda-1)}\left(u^{2}+\varphi^{2}\right)-H^{2}
$$

into the equation for $\dot{H}$ and omitting equation on dynamics of $a$ leads the following set of equations:

$$
\begin{aligned}
u & =\dot{\varphi} \\
\dot{u} & =-\frac{2}{3 \lambda-1} \varphi-3 u H, \\
\dot{H} & =\frac{2}{3(3 \lambda-1)}\left(\varphi^{2}-\frac{u^{2}}{2}\right)-2 H^{2},
\end{aligned}
$$

This is a reduced 3 -dimensional subset of $22 \mid 26)$ on variables $(\varphi, u, H)$. If one wants to obtain also dynamics of $a$, he needs to add to this system equation $\dot{a}=a H$ and also the constraint equation (26).

In subsequent considerations we shall focus on a case $k \neq 0$ (when HL corrections play a significant role) and a phase portrait of solution of the system (33) in space of $(\varphi, u, H)$, following similar a similar procedure as that described in ([32, 33] $)$. Reducing dimensionality of phase space enables 3D phase portrait visualizations. Moreover, we will discuss shortly also a case $k=0$, which play a role of a limiting case of $k \neq 0$ dynamics.

We start by rewriting equations $(33) 35)$ in terms of the variables

$$
x \equiv \varphi ; y \equiv \dot{\varphi} ; z \equiv \frac{\dot{a}}{a},
$$

which gives three "evolution" equations

$$
\begin{aligned}
& \dot{x}=y, \\
& \dot{y}=-\frac{2}{3 \lambda-1} x-3 y z, \\
& \dot{z}=\frac{2}{3(3 \lambda-1)}\left(x^{2}-\frac{y^{2}}{2}\right)-2 z^{2},
\end{aligned}
$$

The space of solution of the above dynamical system is a $3 \mathrm{D}$ region of the phase space $(x, y, z)$. This region is bounded by a 2D surface defined by a constraint equation (31) - space of trajectories of a flat universe $(k=0)$. This limiting surface is a double cone, with the upper branch corresponding to expansion and lower one to contraction. Those two branches connect at a point: $(0,0,0)$, which is a critical point (see below). Hence there are no trajectories passing from one branch of the cone to the other. For $k= \pm 1$ all trajectories lie between the branches of this cone. This cone is also a limiting surface for trajectories with large $a$. The further a trajectory lies from this cone, the smaller are the values of $a$ along it.

Moreover, with the value of $\lambda$ varying between 1 and $\infty$, the double cone reduces to the surface $z=0$ when $\lambda=\infty$. Thus for finite values of $\lambda$ the whole dynamics of the system is contained within the double cone $z^{2}=\frac{1}{3(3 \lambda-1)}\left(x^{2}+y^{2}\right)$, and for $\lambda=\infty$ all phase point lay on 
the surface $z=0$. In the latter case the phase dynamics is flat, the Universe is static, and there are no bounce points. Such a situation is not interesting when searching for a bounce. For finite $\lambda$ the qualitative description of the system does not depend on the specific value of this coupling constant, as it corresponds to the angle of the limiting cone and "shrinking" trajectories between double cone's branches. Therefore in order to simplify further calculations we will set the value $\lambda=1$.

The bounce happens when a phase trajectory passes from the region $z<0$ to region $z>0$, intersecting the plane $z=0$. At the crossing point $\dot{z}>0$ must hold. Equation 39 implies that this happens if the crossing point is contained between lines $y=\sqrt{2} x$ and $y=-\sqrt{2} x$ laying on the plane $z=0$. Those lines are the $z=0$ section of an elliptic cone $\frac{1}{6}\left(x^{2}-\frac{y^{2}}{2}\right)-z^{2}=0$, whose interior consists of trajectories with $\dot{z}>0$ (eq. (39)). The area outside this cone is filled with trajectories along which $\dot{z}<0$.

To find the finite critical points we set all right-hand-sides of equations $(37 \sqrt[39]{3}$ to zero. This gives rise to the conditions

$$
x=y=z=0 .
$$

Stability properties of this point are determined by the eigenvalues of the Jacobian of the system (37 39). More precisely, one has to linearize transformed equations $(37 \sqrt{39})$ at each point. Inserting $\vec{x}=\vec{x}_{0}+\delta \vec{x}$, where $\vec{x}=(x, y, z)$, and keeping terms up to 1st order in $\delta \vec{x}$ leads to an evolution equation of the form $\delta \dot{\vec{x}}=A \delta \vec{x}$. Eigenvalues of $A$ describe stability properties at the given point.

At the finite critical point $O=(0,0,0)$, the matrix $A$ has 2 purely imaginary eigenvalues, which implies there are closed orbits in the $x y$-plane encircling the $z$-axis, i.e. point $O$ lays on a center line surrounded by closed orbits. It is interesting to note, that in general case $\lambda \neq 1$ eigenvalues of $A$ are following: $\left(\sqrt{\frac{2}{1-3 \lambda}},-\sqrt{\frac{2}{1-3 \lambda}}, 0\right)$. Hence for $\lambda>1 / 3$ point $O$ lays on a center line, for $\lambda<1 / 3$ it is a saddle. Nonetheless, the latter case is excluded form our considerations, as we explained in the introduction.

To find critical points that occur at infinite values of the parameters we rescale the infinite space $(x, y, z)$ into a finite Poincaré sphere by means of the coordinate change:

$$
\begin{aligned}
& x=\frac{X}{1-r}, \\
& y=\frac{Y}{1-r}, \\
& z=\frac{Z}{1-r},
\end{aligned}
$$

where

$$
\begin{aligned}
X & =r \sin \theta \cos \varphi, \\
Y & =r \sin \theta \sin \varphi, \\
Z & =r \cos \theta, \\
r^{2} & =X^{2}+Y^{2}+Z^{2} .
\end{aligned}
$$

We shall use both Cartesian coordinates $(X, Y, Z)$ and spherical ones: $(r, \theta, \varphi)$. We also rescale the time parameter $t$ by defining new time parameter $T$ such that: $d T=d t /(1-r)$. In these coordinates our phase space is contained within a sphere of radius one - infinity corresponds to $r=1$.

This is a conformal transformation, hence the limiting cone for phase trajectories is $Z^{2}=$ $\frac{1}{6}\left(X^{2}+Y^{2}\right)$; all physical trajectories are contained within this cone. Bounce points are located on the plane $Z=0$ within the region bounded by lines $Y=\sqrt{2} X$ and $Y=-\sqrt{2} X$. The region containing trajectories with $\dot{Z}>0$ (i.e. with $H$ increasing) is bounded by an elliptic cone $\frac{1}{6}\left(X^{2}-\frac{Y^{2}}{2}\right)-Z^{2}=0$. 


\begin{tabular}{|c|c|c|c|} 
Point & $\varphi$ & $\theta$ & Stability \\
\hline S1 & $\arcsin \sqrt{2 / 3}$ & $\pi / 2$ & Saddle line \\
\hline S2 & $\pi-\arcsin \sqrt{2 / 3}$ & $\pi / 2$ & Saddle line \\
\hline S3 & $\pi+\arcsin \sqrt{2 / 3}$ & $\pi / 2$ & Saddle line \\
\hline S4 & $2 \pi-\arcsin \sqrt{2 / 3}$ & $\pi / 2$ & Saddle line \\
\hline A1 & 0 & $\arccos \frac{\sqrt{7}}{7}$ & Attracting line \\
\hline R1 & 0 & $\pi-\arccos \frac{\sqrt{7}}{7}$ & Repelling line \\
\hline R2 & $\pi / 2$ & $\arccos \frac{\sqrt{7}}{7}$ & Repelling node \\
\hline A2 & $\pi / 2$ & $\pi-\arccos \frac{\sqrt{7}}{7}$ & Attracting node \\
\hline A3 & $\pi$ & $\arccos \frac{\sqrt{7}}{7}$ & Attracting line \\
\hline R3 & $\pi$ & $\pi-\arccos \frac{\sqrt{7}}{7}$ & Repelling line \\
\hline R4 & $3 \pi / 2$ & $\arccos \frac{\sqrt{7}}{7}$ & Repelling node \\
\hline A4 & $3 \pi / 2$ & $\pi-\arccos \frac{\sqrt{7}}{7}$ & Attracting node
\end{tabular}

Table 1. The properties of the infinite critical points.

After Poincaré transformation, equations $(37 \sqrt[39]{39}$ take the following form, written in the spherical coordinates $(r, \theta, \varphi)$ :

$$
\begin{aligned}
r^{\prime}= & \frac{(r-1) r^{2}}{48} \cos \theta[82+14 \cos 2 \theta-42 \cos 2 \phi \\
& +21 \cos 2(\theta-\phi)+21 \cos 2(\theta+\phi)], \\
\theta^{\prime}= & \frac{1}{24} r \sin \theta(5+7 \cos 2 \theta)(1+3 \cos 2 \phi), \\
\phi^{\prime}= & r-1-3 r \cos \theta \cos \phi \sin \phi .
\end{aligned}
$$

The form of the above equations is similar to the ones obtained in [32, 33]. Taking limit $r=1$ and putting r.h.s. of equations for $\theta^{\prime}$ and $\phi^{\prime}$ to zero, we find 12 solutions for $\theta, \phi$ at the Poincaré sphere, shown in the Table 1 . As we can see, there are 4 saddle points (more precisely saddle lines with end points at $\left.S_{1}, S_{2}, S_{3}, S_{4}\right)$. In the contracting part of the phase portrait $(z<0)$ there are two attracting nodes $A_{2}$ and $A_{4}$ and two repulsing lines starting at $R_{1}$ and $R_{3}$. Hyperbolic areas near the nodes are bounded by repulsing lines which play role of separatrices. The expanding part is a mirror ("reversed in time") of the contracting one. Stability properties of those point does not depend on the value of parameter $\lambda$, unless it is in the range $(1 / 3, \infty)$.

Stability properties of infinite critical points are described in the Table 1, their position in 3D phase space, on a Poincaré sphere, is shown in Fig. 1.

\subsection{Trajectories}

When spatial curvature $k=0$ then phase trajectories lay on the limiting cone $\tilde{z}^{2}=\frac{1}{6}\left(X^{2}+Y^{2}\right)$, as shown in the Figure 2. In the contracting part all trajectories start winding around $z$-axis, then some of them end at attracting node $A_{2}$, some at $A_{4}$. Those two families are separated by repelling lines with end-points at $R_{1}$ or $R_{3}$, acting as separatrices. Expanding part is a mirror reflection with time reversed of the contracting part.

Trajectories of non-flat universes lay inside the limiting cone of flat space. In the contracting part of the diagram $(Z<0)$, trajectories start spiraling outside from circles around the $Z$-axis. There are two families of such trajectories, separated by repelling lines ending at $R_{1}$ and $R_{3}$. In each family, there are two possible scenarios for subsequent evolution. The first one, shown in Figure $3 \mathrm{a}$, is to end at an attractor node $\left(A_{2}\right.$ or $\left.A_{4}\right)$, which also lays in the contracting part of the phase diagram. On the way between $O$ and $A_{2}$ or $A_{4}$, a trajectory may go up through $Z=0$ surface, undergoing a bounce there, and then recollapse, crossing the $Z=0$ plane again, or go straight to the attractor node, without bounce. In either case, the end is a Big Crunch. 


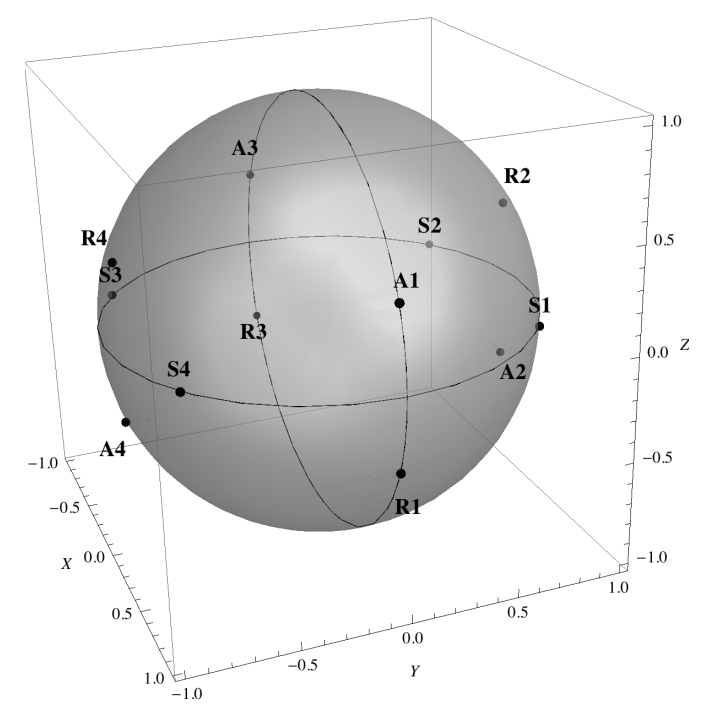

Figure 1. Infinite critical points located on a Poincaré sphere

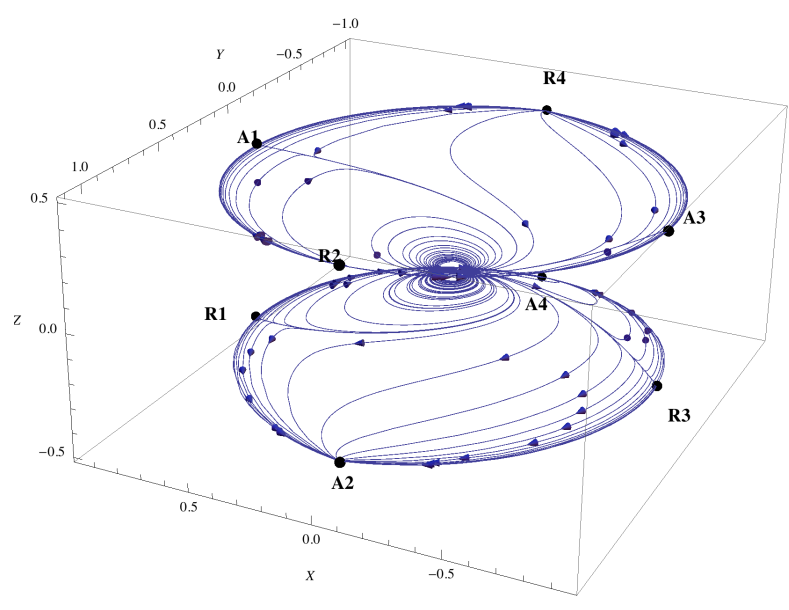

Figure 2. Phase trajectories for flat HL universe

The second scenario is shown on the Figure 3c. Here, after some oscillations and $H$ decreasing, the trajectories reach an attractor - a repelling line (that ends either at $R_{1}$ or $R_{3}$ ), along which they move until $\dot{H}=0$. Then they rapidly go up, crossing the $Z=0$ (i.e. $H=0$ ) plane, undergoing a bounce. After that, and after a period of accelerated expansion, they reach another attractor an attracting line laying in the expanding part, with endpoint at either $A_{1}$ or $A_{3}$. Along this line trajectories approach the $Z$-axis, winding around it. A subcase of this scenario is shown on the Figure $3 \mathrm{e}$, where trajectories do not go through accelerated contraction and expansion, but cross the $Z=0$ surface during oscillations around the $Z$-axis. This is in fact the scenario described in 22 .

Trajectories may also start at repelling nodes $R_{2}$ or $R_{4}$ in the expanding part of the diagram. At those points $H=\infty$, i.e. there is a Bing Bang there. After that and a period of extreme, but with decreasing rate, expansion, there are again two possible scenarios. One is shown on the Figure $3 \mathrm{~b}$, where trajectories reach an attracting line and end up winding around the $Z$-axis. Before that, some of them collapse, crossing the $Z=0$ plane, then slow down and finally stop contraction culminating in a bounce. Others show only slow expansion, without crossing $Z=0$. 


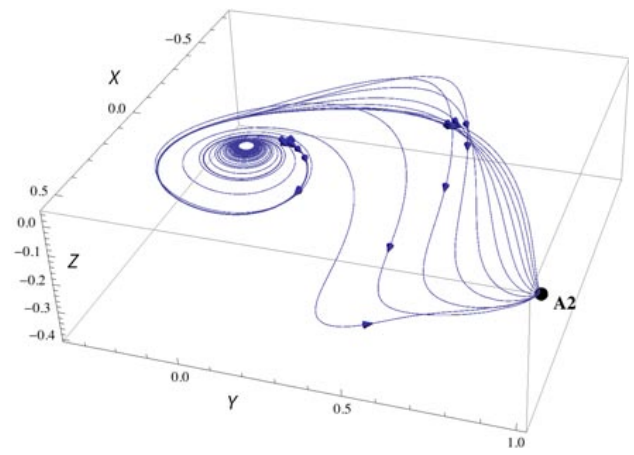

(a)

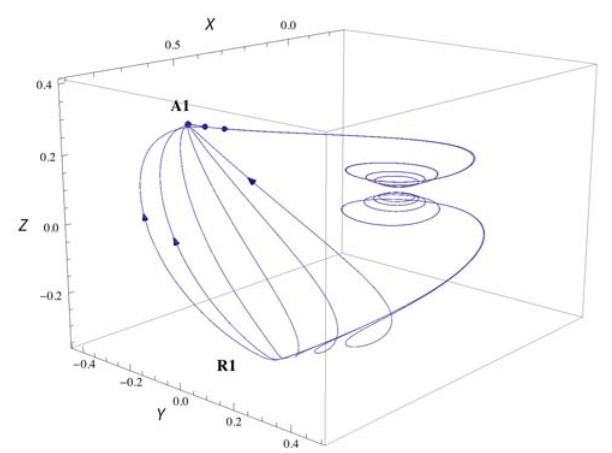

(c)

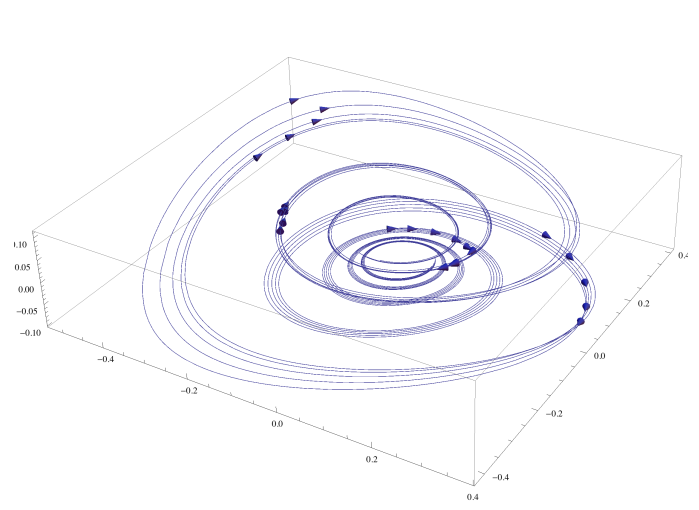

(e)

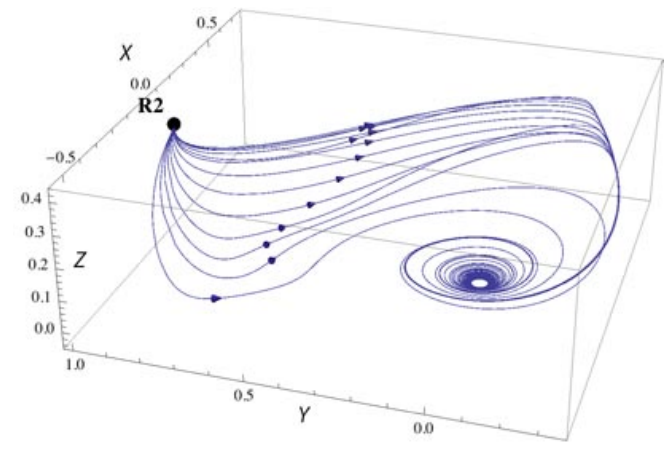

(b)

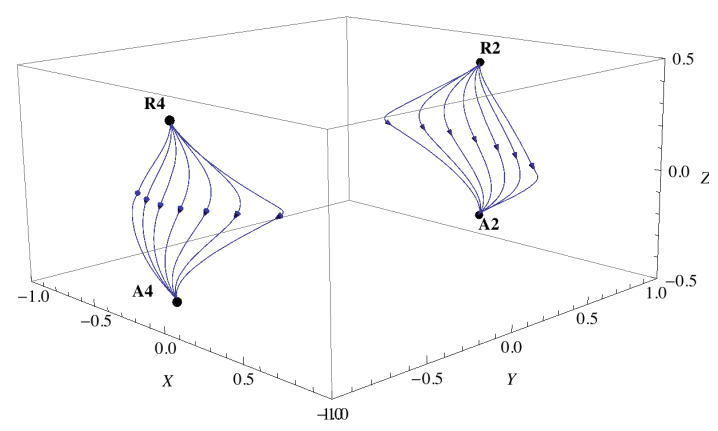

(d)

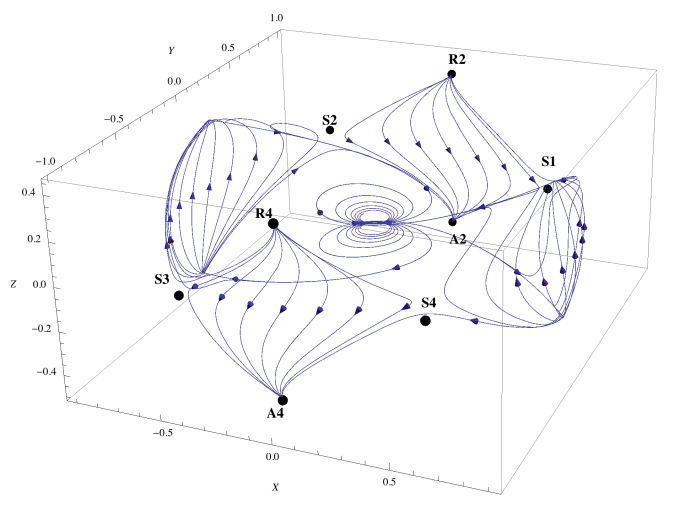

(f)

Figure 3. Different types of phase trajectories for a non-flat Hořava-Lifshitz universe

The last scenario is shown on the Figure $3 \mathrm{~d}$. Trajectories start at Big Bang points $R_{2}$ or $R_{4}$, and after a period of slowing down expansion, reach a turning point and start accelerated contraction, ending at Big Crunch points $A_{2}$ or $A_{4}$.

For better visualization we have gathered some described families of trajectories Figure $3 \mathrm{f}$.

Special attention has to be paid to circular motion around z-axis. As stability properties of the point $(0,0,0)$ and constraint equation $(26)$ suggest, there may exist closed circular orbits laying on a $Z=0$ plane $(H=0)$. But they cannot. Equation 39 does not allow for this, as $\dot{Z}=0$ is fulfilled only on a class of curves laying on $X^{2}-\frac{X^{2}}{2}-6 Z^{2}=0$, i.e. on a surface of the elliptic cone mentioned before. Yet numerical simulations exhibit oscillating solutions, such as $X^{2}+Y^{2}=$ const. and $Z$ oscillated around zero, $\dot{Z}>0$ between lines $Y=\sqrt{2} X$ and $Y=-\sqrt{2} X$, $\dot{Z}<0$ outside this region. Such a trajectory resembles deformed circle. These solutions appear 
for sufficiently small $X$ and $Y$, for larger values of $X$ and $Y$ numerical simulations show slow decreasing of the radius of this "circle" .

Finally, note that except for the special solution shown in Figure 3e - which is the bounce described by Brandenberger [22, there are also other types of bounces. One, probably the most interesting, is shown in Figure 3c. Here a big existing universe slowly starts to contract, but later on the contraction becomes exponential, until a bounce is reached and an exponential expansion begins, which finally slows down. Another type of bounce, shown on the Figure 3a, happens again when a big universe slowly starts contracting, stops and goes through an expanding phase for a while, then recollapses and ends at Big Crunch. The last one, shown on the Figure 3b, happens during a transition from a Big Bang to a quasi stationary final stage (with $H$ slowly decreasing), however with a bounce on the way.

Trajectories shown on the figures discussed above are numerical solutions of the equations 48 50. To find different bounce scenarios we investigated initial conditions: $\theta=\pi / 2 \pm 0.01$, $\phi=i \frac{\pi}{20}(i=1 \ldots 20)$, each for $r=j / 10(j=1 \ldots 9)$ and $r=0,9+j \cdot 0.01$; time in range $[-20,20]$. This procedure picked up the classes of trajectories discussed above. In general it may not be exhaustive in the sense that qualitatively different behavior of solutions may be possible. However it is sufficient for the purpose of understanding how bouncing scenarios emerge here due to the specific modification of general relativity which appears in Hořava's theory.

\section{Discussion and conclusions}

In this paper we have investigated the cosmological bounce in Hořava-Lifshitz gravity. Using a $3 \mathrm{D}$ flow visualization technique we have found that phase portraits in the considered theory have a different structure than in standard cosmology. Comparing to results from the paper [33], we can see that here are additional repellers $\left(R_{1}\right.$ and $\left.R_{2}\right)$ in the contracting part of a phase space, and mirror attractors in the expanding part. Their presence allows the existence of a bounce, because now there are possible new families of trajectories, starting at additional repellers in the contracting part, and possibly ending at new attractors in expanding part, or surrounding the $(0,0,0)$ point, which is now a center, compared to saddle in standard cosmology. Those are realizations of the bounce. One of them is the solution with oscillatory behavior described in (22]); there are however additional possibilities. The most interesting one contains a period of rapid contraction, and - after a bounce - a period of rapid expansion, what may fit inflationary scenario.

Nevertheless there are still initial conditions which lead to a Big Crunch, as shown on the Figures 3a and 3d, or which start at initial singularity (Fig. 3b and 3d). Hence the existence of a bounce is not generic for Hořava theory and depends on initial conditions.

Another interesting class of solutions consists of quasi stationary universes. These solutions are described in phase space by closed orbits, winding around the critical point $(0,0,0)$ - a center. All trajectories in the neighborhood of this point end up as closed orbits, "deformed circles". Equations of motion do not allow for closed orbits laying on $Z=$ const. plane, resulting in slight deformation of the circular orbits. The values of $H$ oscillate around stationary stage, for sufficiently small values of $\varphi$ and $\dot{\varphi}$. Values of scale parameter $a$ during this evolution are much bigger than the regime for which our simplifications are valid. Therefore this behavior is not a feature of HořavaLifshitz theory, but of cosmologies with modified equations of motion, i.e. with the additional term $\sim 1 / a^{4}$ in the Friedmann equations. Still, presence of this term leads to a different solution than induced by a negative potential as in [33], due to different stability properties of finite critical points there.

The visualizations described in this paper describe the dynamics of Hořava-Lifshitz universe with vanishing cosmological constant $\Lambda$, or HL universe with non-zero Lambda in the region of small scale factor $a$. Even in such slightly limited framework they answer the question of possible scenarios realizing a bounce, and whether it is generic for the theory or not. It appears not, as we have found solutions leading to a Big Crunch, or starting at Big Bounce, both staying within the regime of small $a$. There is also an interesting possibility of quasi stationary, oscillating universe, 
existence of which is clearly implied by dark radiation term in Friedmann equations.

Finally, it is worth stressing that the analysis presented here should be applicable to other theories which lead to modifications of the Friedmann equations.

\section{Acknowledgements}

I would like to thank Michał Spaliński for fruitful discussions.

This work has been supported by the Polish Ministry of Science and Higher Education grant PBZ/MNiSW/07/2006/37.

\section{References}

[1] Khoury J, Ovrut B A, Steinhardt P J and Turok N 2001 Phys. Rev. D 64123522 arXiv:hep-th/0103239; Buchbinder E I, Khoury J and Ovrut B A 2007 Phys. Rev. D 76123503 (2007) arXiv:hep-th/0702154.

[2] Steinhardt PJ and Turok N 2002 Science 296. no. 55721436 arXiv:hep-th/0111030; Steinhardt PJ and Turok N 2002 Phys. Rev. D 65126003 arXiv:hep-th/0111098; Steinhardt PJ and Turok N 2002 Phys. Rev. D 66 101302 astro-ph/0112537.

[3] Kallosh R, Kofman L and Linde A D 2001 Phys. Rev. D 64123523 arXiv:hep-th/0104073.

[4] Ashtekar A, Pawlowski T and Singh P 2006 Phys. Rev. Lett. 9614130 arXiv:gr-qc/0602086

[5] Ashtekar A, Pawlowski T and Singh P 2006 Phys. Rev. D 73124038 arXiv:gr-qc/0604013

[6] Ashtekar A, Pawlowski T and Singh P 2006 Phys. Rev. D $\mathbf{7 4} 084003$ arXiv:gr-qc/0607039.

[7] R. Maartens 2004 Living Rev. Rel. 77 arXiv:gr-qc/0312059.

[8] Randall L and Sundrum R 1999 Phys. Rev. Lett. 834690

[9] Novello M and Perez S E 2008 Phys. Rept. 463 127-213 arXiv:0802.1634 [astro-ph]].

[10] Horava P arXiv:0811.2217 [hep-th]].

[11] Horava P 2009 JHEP 0903020 arXiv:0812.4287 [hep-th]].

[12] Horava P 2009 Phys. Rev. D 79084008 arXiv:0901.3775 [hep-th]].

[13] Horava P arXiv:0902.3657 [hep-th]].

[14] G. Calcagni 2009 JHEP 0909112 arXiv:0904.0829 [hep-th]].

[15] Kiritsis E and Kofinas G. 2009 Nucl. Phys. B 821467 arXiv:0904.1334 [hep-th]].

[16] Saridakis E N arXiv:0905.3532 [hep-th]].

[17] Nastase H arXiv:0904.3604 [hep-th]].

[18] Mukohyama S, Nakayama K, Takahashi F and Yokoyama S arXiv:0905.0055 [hep-th]].

[19] Minamitsuji M arXiv:0905.3892 [astro-ph]].

[20] Wang A and Wu Y 2009 JCAP 0907012 arXiv:0905.4117 [hep-th]].

[21] Takahashi T and Soda J 2009 Phys. Rev. Lett. 102231301 arXiv:0904.0554 [hep-th]].

[22] Brandenberger R arXiv:0904.2835 [hep-th]].

[23] Charmousis C, Niz G, Padilla A and Saffin P M 2009 JHEP 0908070 arXiv:0905.2579 [hep-th]].

[24] Li M and Pang Y 2009 JHEP 0908015 [arxiv: 0905.2751[hep-th]].

[25] Sotiriou T P, Visser M and Weinfurtner S 2009 JHEP 0910033 arXiv:0905.2798 [hep-th]].

[26] Bogdanos C and Saridakis E N arXiv:0907.1636 [hep-th]].

[27] Carloni S, Elizalde E and Silva P J arXiv:0909.2219 [hep-th]].

[28] Leon G and Saridakis E N 2009 JCAP 0911006 arXiv:0909.3571 [hep-th]].

[29] Binétruy P, Deffayet C, Ellwanger U and Langlois D 2000 Phys. Lett. B 477285 hep-th/9910219.

[30] Bogdanos C and Saridakis E N 2010 Class. Quant. Grav. 27075005 arXiv:0907.1636.

[31] Lu H, Mei H and Pope C N arXiv:0904.1595 [hep-th]].

[32] Felder G N, Frolov A and Kofman L. 2002 Class.Quant.Grav. 192983 arXiv:hep-th/0112165.

[33] Felder G N, Frolov A, Kofman L and Linde A 2002 Phys. Rev. D 66023507 arXiv:hep-th/0202017. 